\title{
The Modified Procedure of Totally Tubeless PNL
}

\author{
Andriy I. Sahalevych, Roman V. Sergiychuk, Vladislav V. Ozhohin, Andriy Yu. Khrapchuk, \\ Yaroslav O. Dubovyi, Olexander S. Frolov \\ Shupyk National Healthcare University of Ukraine, \\ 9 Dorohozhytska Str., 04112 \\ Ukraine
}

Received: May 27, 2021. Revised: November 9, 2021. Accepted: December 13, 2021. Published: January 3, 2022.

\begin{abstract}
Mini percutaneous nephrolithotomy (mPNL) is a standard treatment for kidney stones larger than 1.5 $\mathrm{cm}$, with the placement of a nephrostomy drainage at the end of it, which is considered the standard procedure, but tubeless/ totally tubeless mPNL techniques reduce postoperative discomfort in patients and shorten hospital stays. The aim of article was to compare the efficacy and safety of our proposed modified method of totally tubeless mPNL with control of the parenchymal canal, with existing methods of tubeless/totally tubeless mPNL. Novelty of the study presented by modified method of totally tubeless mPNL. During the period from 2018 to 2020 we performed $486 \mathrm{mPNL}$ were performed in our clinic in total, among which $63(12.9 \%)$ patients underwent tubeless PNL. Patients whose surgeries ended with using tubeless techniques were divided into three groups: Group I - 22 patients who had tubeless mPNL (with ureteral stent), Group II (20 patients) - totally tubeless MPNL with a safety thread (the proposed procedure), Group III (21 patients) - totally tubeless mPNL. In all three groups, the access point was most often made through the lower group of renal calyces: Group I - $12(54.5 \%)$, Group II - 14 (70.0\%), Group III - $13(61.9 \%)$; then through the middle calyx: Group I - 8 (36.4\%), Group II - $6(30.0 \%)$, Group III - 7 (33.3\%); and the upper calyx: Group I - $2(9.1 \%)$, Group II - 0\%, Group III - $1(4.8 \%)$, no differences in the distribution of access points between groups were found $(p=0.67)$. There were no differences in the distribution of tract sizes between the groups $(p=0.95)$ with tract dilatation to $16.5 / 17.5 \mathrm{Fr}$ was performed most often: Group I - $12(54.5 \%)$, in Group II - $11(55.0 \%)$ and Group III - $11(52.4 \%)$. The mean duration of surgery in Group I was 83.0 $\pm 22.9 \mathrm{~min}$, in Group II $-\mathbf{7 4 . 9 \pm 1 3 . 6} \mathrm{min}$, in Group III - 72.6 $\pm 12.0 \mathrm{~min}(\mathrm{p}=\mathbf{0 . 4 7})$. This study confirms the high effectiveness of totally tubeless mPNL. The
\end{abstract}

proposed modification to perform totally tubeless $\mathrm{mPNL}$ allows you to have permanent postoperative control over the parenchymal channel and in case of postoperative bleeding it enables you to immediately insert nephrostomy drainage through the safety thread. Study contributes to practical methods as an intermediate step for surgeons who are considering transition to a totally tubeless PCNL technique.

Keywords-percutaneous nephrolithotomy; tubeless PNL; epidural anesthesia; ureteral stent; kidney stones.

\section{INTRODUCTION}

P ercutaneous nephrolithotomy (PNL) was first performed in 1976 and since then it has remained the preferred method for removal of kidney stones larger than 1.5-2.0 cm [1$3]$. For the first time, the totally tubeless nephrolithotomy technique was proposed by one of the founders of percutaneous surgery, J. Wickham et al., who back in 1984 presented the results of 100 successful totally tubeless PNLs with neither a nephrostomy tube nor a ureteral catheter inserted [4]. Unfortunately, this technique was not widely adopted until this century. The reason for not using tubeless techniques was the high risks of developing early postoperative bleeding, requiring open surgery or embolization of damaged vessels of the renal parenchyma. Even today, with the proven PNL technique, intra-, and postoperative bleeding remains one of the most formidable complications of PNL, the level of which is up to $7.0 \%$ [5]. The second point of the small number of tubeless PNL supporters was the risk of urinary leakage, requiring repeated urinary tract drainage.

In 2003, Yew et al. [6] presented a modification of tubeless PNL by placing a JJ (urethral) stent with a thread attached to the distal tip exiting the urethra, and removal of the stent was performed without cystoscopy simply by pulling the threads. In 2006, Shpall et al. [7] developed a new tubeless PNL 
technique with intraoperative placement of the ureteral stent in a reverse orientation with threads affixed to the proximal tip of the stent and exiting through the percutaneous tract in the flank, which allowed stent removal by pulling the percutaneous tip of the thread. This Shpall's technique allows you to remove the ureteral stent not only without the use of repeated cystoscopy, but it is very important to maintain control over the parenchymal canal and, if necessary, insert a nephrostomy drainage, without performing repeated operations.

In an effort to minimize trauma to the kidneys and intrarenal vessels, the last decade has been marked by the wide adoption of mini percutaneous nephrolithotomy ( $\mathrm{mPNL}$ ), with decrease in size of external sheath to $<22 \mathrm{Fr}[8 ; 9]$. Several metaanalyses showed that stone-free rate (SFR) when performing mPNL were equal to the rate of standard PNL, while reducing the length of hospitalization, postoperative pain, and the need for analgesics [10-13]. This result is obtained due to the fact that the interventions performed during operations are practically the same for both methods, and the main difference lies in the size of the instruments. As postoperative hemorrhage remains a known complication, urologists who commence carrying out PNL, even when they are able to perform tubeless surgery, often do not do it, for fear of bleeding in the postoperative period due to lack of control over the percutaneous tract [14-18]. Since one of the indications for tubeless /totally tubeless PNL is the absence of both intraoperative bleeding and the absence of the risk of postoperative bleeding.

The concerns and limitations of above-mentioned techniques served as an impetus to develop new modifications of existing techniques of totally tubeless mPNL, in which it would be possible to maintain permanent control over the percutaneous tract in the early postoperative period without requiring an indwelling nephrostomy tube or ureteral stent.

\section{MATERIALS AND METHODS}

During the period from January 2018 to December 2020, $486 \mathrm{mPNLs}$ were performed in our clinic in total, among which $81(16.6 \%)$ patients whose mPNL was completed by tubeless PNL techniques, 63 (12.9\%) of which were included in the study. The inclusion criteria were: age of patients over 18 years, surgery with one percutaneous access tract, renal calculus size $<30 \mathrm{~mm}$. Patients who had renal anomalies, staghorn calculi, obstructive symptoms of the lower urinary tract, hydronephrosis, anatomically or functionally solitary kidney, acute inflammation of the kidneys, pyuria, acute or chronic renal failure, coagulopathy, and intraoperative bleeding or perforation of the renal collecting system were excluded from this study.

Patients were divided into three groups. The first group (Group I) included 22 patients who underwent tubeless mPNL (with ureteral stent), the second group (Group II) - 20 patients after totally tubeless mPNL with a safety thread (our proposed procedure), the third group (Group III) included 21 patients after totally tubeless mPNL. For an objective assessment, all surgeries were performed by the same surgeon with more than 20 years of PNL experience. Demographics and stone characteristics of each group under study are given in Table 1. There were no statistically significant differences in age and sex, location and size of stones $(\mathrm{p}>0.05)$. Correlation between the size of the stone and its localization was found. Stones up to $10 \mathrm{~mm}$ are more frequently localized medially, and those larger than $10 \mathrm{~mm}$ are somewhat more common in the pyelon.

Table 1. Demographics and Stone Characteristics of Patients

\begin{tabular}{|c|c|c|c|c|}
\hline & $\begin{array}{c}\text { Tubeless mPNL, Group } \\
1(\mathrm{n}=\mathbf{2 2})\end{array}$ & $\begin{array}{c}\text { Totally tubeless mPNL, } \\
\text { Group } 2(n=20)\end{array}$ & $\begin{array}{c}\text { Totally tubeless mPNL, } \\
\text { Group } 3(n=21)\end{array}$ & P Value* \\
\hline Age (y) & $53.5 \pm 12.4$ & $58.0 \pm 9.9$ & $55.1 \pm 13.1$ & 0.48 \\
\hline \multicolumn{5}{|l|}{$\operatorname{Sex}(n, \%)$} \\
\hline Male & $10(45.5)$ & $9(45.0)$ & $11(52.4)$ & \multirow{2}{*}{0.87} \\
\hline Female & $12(54.5)$ & $11(55.0)$ & $10(47.6)$ & \\
\hline \multicolumn{5}{|c|}{ Stone laterality $(\mathrm{n}, \%)$} \\
\hline Left kidney & $12(45.5)$ & $9(45.0)$ & $11(52.4)$ & \multirow{2}{*}{0.813} \\
\hline Right kidney & $10(54.5)$ & $11(55.0)$ & $10(47.6)$ & \\
\hline \multicolumn{5}{|c|}{ Locations of the stones $(n, \%)$} \\
\hline Upper calyx & $1(4.5)$ & - & $1(4.8)$ & \multirow{5}{*}{0.60} \\
\hline Middle calyx & $1(4.5)$ & $2(10)$ & $2(9.5)$ & \\
\hline Lower calyx & $3(13.6)$ & $3(15)$ & $4(19.0)$ & \\
\hline Pelvis & $12(54.5)$ & $14(70)$ & $14(66.7)$ & \\
\hline Upper ureter & $4(18.2)$ & $1(5)$ & - & \\
\hline Stone size (mm) & $22.0 \pm 3.5$ & $22.2 \pm 4.1$ & $21.7 \pm 4.9$ & 0.92 \\
\hline
\end{tabular}

Note: *Statistically significant, if $\mathrm{p}<0.05$, , $y$-year, $n$-sample size, $m m-$ millimeters.

All procedures were performed under regional epidural anesthesia (Bupivacaine $20 \mathrm{ml} / 50 \mathrm{mg}$ ). Initially, retrograde catheterization of the kidney was done with a 6 Fr ureteteral catheter. Patients were then repositioned prone position. Under combined ultrasonography and fluoroscopy guidance we carried out puncture access to the renal collecting system with serial dilatation with plastic and metal dilators until a $15 / 16$ or 16.5/17.5 Fr nephroscope tube was installed, followed by the use mini-nephroscope (Karl Storz, Tuttlingen, Germany). Stone fragmentation was performed using Ho:YAG laser 
Medilas H (Dornier) and fragments were removed. Antegrade pyelography was then performed to assess the integrity of the renal cavity system and to evaluate the upper urinary tract patency. In case of the absence of the above-mentioned complications, a wire guide was inserted through the nephroscope to the renal cavity system, and the nephroscope with the tube was pulled out, inspecting the percutaneous tract. If there was no bleeding, it was decided to perform surgery by the method of totally tubeless mPNL. Otherwise, nephrostomy drainage (Foley catheter 12Fr, open end) was placed, and that group of patients was excluded from the study. In the case of mucosal edema of the uretero-pelvic junction or the upper third of the ureter, we placed antegrade ureteral JJ-stent, followed by the decision to perform tubeless $\mathrm{mPNL}$.

The proposed modified procedure of totally tubeless mPNL with a safety thread was carried out after the main stage of
mPNL. A safety thread (Prolene No.0, length $150 \mathrm{~cm}$ ) was inserted into in the proximal (urethral) lumen of the ureteral catheter, which was passed into the renal cavity system; the distal tip of the thread, under the visual guidance of the nephroscope, was grasped with forceps and removed through the nephroscope tube outwards (Fig. 1, 2). During utilization of this technique in $90 \%$ of patients, no adverse events were noter in the postoperative period, and the safety thread was removed on postoperative day. The ureteral catheter was then pulled outwards by the urethral (proximal) end, so that the urethral portion of the safety thread remained outside the urethra. The nephroscope sheath is removed while visually inspecting the tract for bleeding with the distal portion of the safety thread remained outside the percutaneous tract in the flank.

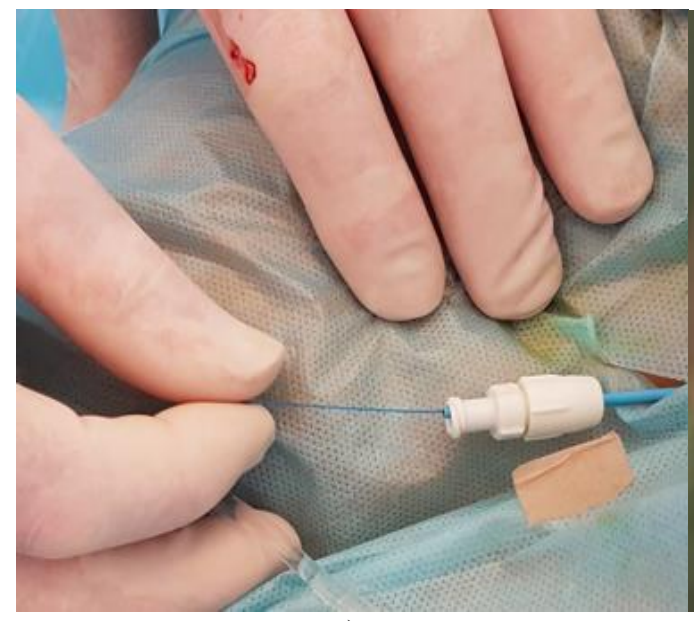

a)

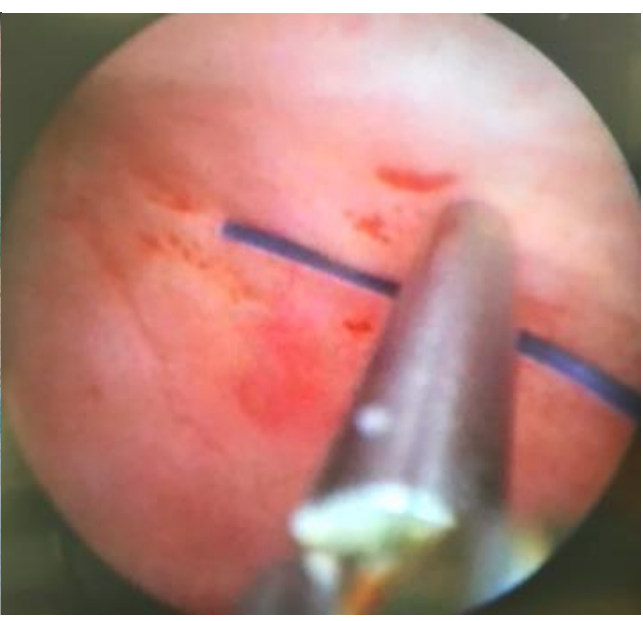

b)

Fig. 1. Insertion of a safety thread: a) - insertion of a safety thread into the proximal tip of the ureteral catheter; b) - grasping a safety thread in the renal cavity system

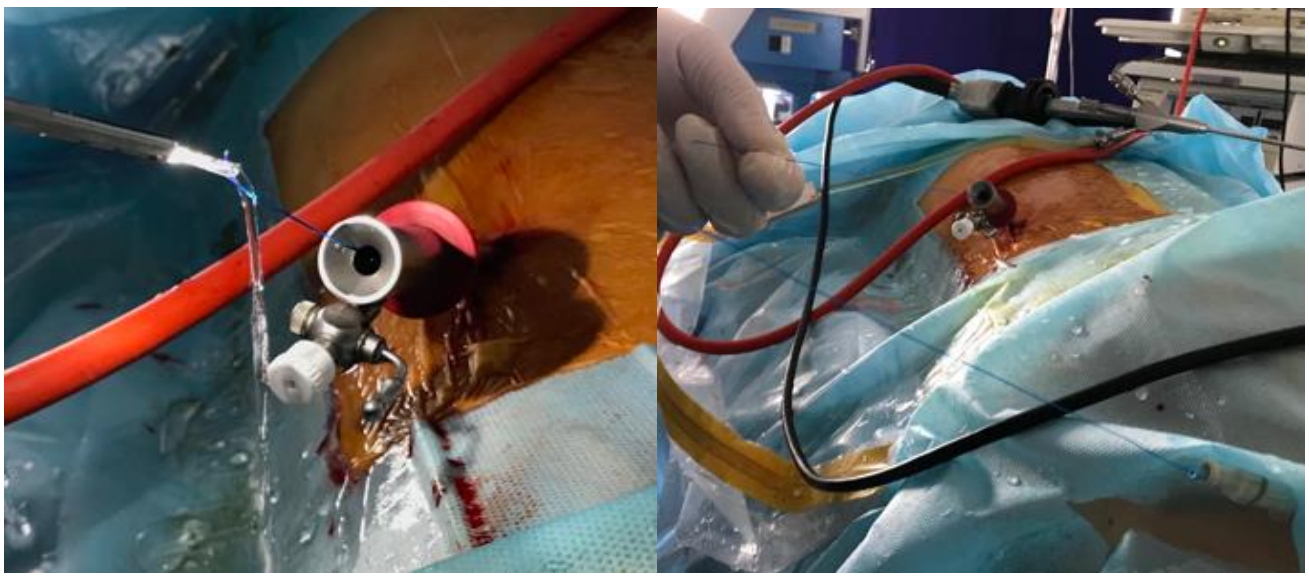

a)

b)

Fig. 2. Pulling the tips of a safety thread outwards: a) - removal of the distal tip of the thread outwards; $b$ ) - the proximal and distal tips of the thread are outwards

If no bleeding was seen, the skin incision is closed and the distal end of the safety thread is fixed to the skin, while the urethral end of the safety thread is affixed to a Foley catheter with adhesive tape (Fig. 3, 4). The technique mimics a totally tubeless mPCNL, with a safety suture thread allowing maintenance of control over the percutaneous tract and the 
collecting system. In case of bleeding in the early postoperative period, it is possible to place a nephrostomy tube along the suture thread for hemostasis and drainage of the collecting system. The technique may be helpful as an intermediate step for surgeons who are considering transition to a totally tubeless PCNL technique. The patient is monitored overnight, and if no post-operative complications occurred an ultrasound scan of the urinary system is performed to ensure no residual concretions or blood clots are seen in the renal collecting system. Following this, the distal portion of the safety thread is cut close to the skin, and the safety thread is pulled out along with a Foley catheter.

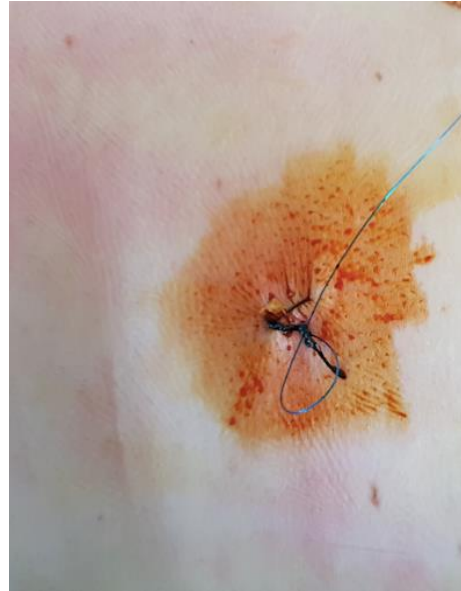

a)

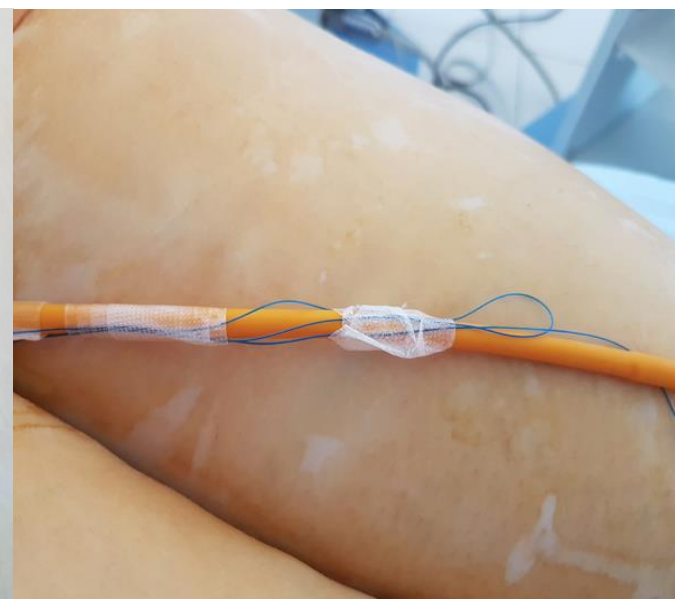

b)

Fig. 3. Fixation of the thread tips: a) - The percutaneous tip of a safety thread is fixed to the skin; b) - the distal tip of the safety thread is fixed to a Foley catheter

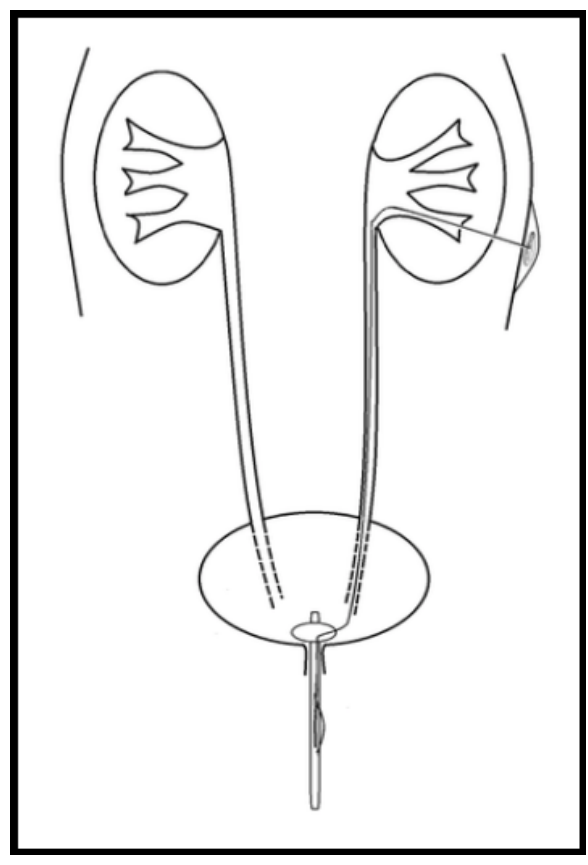

Fig. 4. Schematic image of inserting a safety thread while performing modified totally tubeless PNL

In case of bleeding, upper tract obstruction, or fever, the safety thread can be used as a guide wire. This is accomplished by pulling the safety thread at both ends and either inserting a nephrostomy tube into the distal side of the safety thread to stop bleeding, or inserting a JJ stent either into safety thread in a retrograde or antegrade fashion. In the postoperative period, we assessed SFR, the presence of postoperative complications, the need for analgesia, the number of postoperative bed-hours, and the need for second-look interventions. Pain scores in patients were quantified on an international visual analog scale, according to which pain intensity is assessed in certain terms using a 0-10 ranking scale.

Statistical data analysis was performed using software EZR v. 1.35 ( $\mathrm{R}$ statistical software version 3.4.3, R Foundation for Statistical Computing, Vienna, Austria) [19-22]. To represent quantitative indicators, the mean value (loverline $\{X\}$ ) and standard deviation $( \pm$ SD) were calculated in case of normal distribution law of the indicator or median $(\mathrm{Me})$ and the interquartile range (IQR) in case of distribution law unlike a normal one. The normality distribution was checked according to the Shapiro-Wilk test. Frequency (\%) was calculated to represent qualitative indicators. When comparing quantitative indicators in three groups, Analysis of Variance and the Scheffe test (in case of a normal distribution law) or nonparametric Kruskal-Wallis test were used, and Dunn's multiple comparison test (in case of a distribution law unlike a normal one) was used for pairwise posterior comparisons. The chisquare test was used to compare qualitative data. Criteria with two-sided critical area were used, the critical significance level was set at 0.05 .

\section{RESULTS}

Clinical and demographic variables are summarized in Table 1 and intraoperative parameters of each group are presented in Table 2. Studies presented that mini-PCNL can achieve the same STR as the standard PCNL, but with small 
scale tools. When the smaller UMP and micro-PCNL were introduced, they suffered from decreased visualization, but both techniques' main problem was fragment clearance. The new thulium fiber lasers seem to be able to fragment stones into tiny fragments easy to irrigate and suction even with smaller scopes and sheaths, perhaps partially solving this problem in the future.

The most recent advancement is the super-mini PCNL (SMP) that uses an 8.0-French nephroscope and a specially designed irrigation-suction sheath with a built-in handle. The new SMP generation modification of the technique was developed to overcome the limited irrigation problem, using the same nephroscope and an innovative sheath that provides an inflow irrigation through a special channel in the sheath itself, thus providing continuous irrigation without sacrificing one of the working channels.

SMP also provides a new "negative pressure" stone clearance mechanism, which uses the hydrodynamic properties of the system. This simultaneously overcomes the disadvantages of both UMP and micro-PCNL, while also maintaining low intra renal pressure during the procedure.

A recent multicenter study for the treatment of $1-2 \mathrm{~cm}$ lower pole calculi compare SMP to RIRS, demonstrating superior stone free rate with SMP and similar complication rates [10]. This places SMP in a unique position, bridging the gap between what we used to conceive as standard PCNL, and RIRS surgery. SMP may have its own role in future guidelines for nephrolithiasis, especially if we incorporate economic considerations as well.

Despite its advantages, SMP still suffers from having a smaller tract size with the inherit limitation to visualization and operative time. To our knowledge, SMP is yet to be widely tested for larger stones or staghorn calculi, making standard PCNL the better option for those patients.

Table 2. Intraoperative Parameters

\begin{tabular}{lcccc}
\hline & $\begin{array}{c}\text { Tubeless mPNL, } \\
\text { Group 1 }(\mathbf{n = 2 2})\end{array}$ & $\begin{array}{c}\text { Totally tubeless mPNL, } \\
\text { Group 2 }(\mathbf{n = 2 0})\end{array}$ & $\begin{array}{c}\text { Totally tubeless mPNL, } \\
\text { Group 3 (n=21) }\end{array}$ & P value* \\
\hline Access location (n, \%) & & & & \\
Upper calyx & $2(9.1)$ & - & $1(4.8 \%)$ & 0.67 \\
Middle calyx & $8(36.4)$ & $6(30.0 \%)$ & $7(33.3 \%)$ & \\
Lower calyx & $12(54.5)$ & $14(70.0 \%)$ & $13(61.9 \%)$ & \\
Dilatation (n, \%) & & & $10(47.6)$ & 0.95 \\
14/15Fr & $10(45.5)$ & $9(45.0)$ & $11(52.4)$ & 0.47 \\
16,5/17,5Fr & $12(54.5)$ & $11(55.0)$ & $72.6 \pm 12.0$ & \\
The duration of surgery (min) & $83.0 \pm 22.9$ & $74.9 \pm 13.6$ & & \\
\hline
\end{tabular}

Note: * Statistically significant, if $p<0.05$., $n$-sample size, $\mathrm{Fr}$-fraction.

There were no perioperative blood transfusions. The mean hemoglobin drop-in Group 1 was $0.20(\mathrm{IQR}=0-0.5) \mathrm{g} / \mathrm{dL}$, in Group II $-0.45(\mathrm{IQR}=0-0.7) \mathrm{g} / \mathrm{dL}$, and in Group III -0.40
$(\mathrm{IQR}=0-0.52) \mathrm{g} / \mathrm{dL}$. The difference between parameters under study was not statistically significant $(\mathrm{p}=0.62)$ (Table 3$)$.

Table 3. Results and complications

\begin{tabular}{|c|c|c|c|c|}
\hline & $\begin{array}{l}\text { Tubeless mPNL, } \\
\text { Group } 1(\mathrm{n}=\mathbf{2 2})\end{array}$ & $\begin{array}{c}\text { Totally tubeless mPNL, } \\
\text { Group } 2(n=20)\end{array}$ & $\begin{array}{c}\text { Totally tubeless mPNL, } \\
\text { Group } 3(n=21)\end{array}$ & $P$ value* \\
\hline $\begin{array}{l}\text { Postoperative hemoglobin drop } \\
\text { (g/dL) }\end{array}$ & 0.20 & 0.45 & 0.40 & 0.62 \\
\hline Blood transfusion & - & - & - & \\
\hline $\begin{array}{l}\text { Postoperative pain score (visual } \\
\text { analog scale } 0-10 \text { ) (24 hours) }\end{array}$ & 3.5 & 1.5 & 2.0 & $<0.001$ \\
\hline $\begin{array}{l}\text { Non-narcotic analgesics } \\
\text { (Dexketoprofen, mg) }\end{array}$ & $52.2 \pm 16.1$ & $28.5 \pm 14.1$ & $27.5 \pm 13.8$ & $<0.001$ \\
\hline $\begin{array}{l}\text { Narcotic analgesics } \\
\text { Complications, } \mathbf{n}(\%)\end{array}$ & - & - & - & \\
\hline Fever $>38 \mathrm{C}$ & $2(9.1 \%)$ & $1(5.0 \%)$ & - & 0.36 \\
\hline Bleeding & 年 & $1(5.0 \%)$ & - & 0.34 \\
\hline $100 \%$ Stone free rate & $21(95.5 \%)$ & $20(100.0 \%)$ & $21(100.0 \%)$ & 0.60 \\
\hline $\begin{array}{l}\text { Second-look interventions, } \mathbf{n} \\
(\%)\end{array}$ & - & 1(5.0) (nephrostomy) & - & 0.34 \\
\hline Postoperative bed-day (hours) & $28.4 \pm 9.5$ & $27.6 \pm 11.7$ & $25.1 \pm 5.2$ & 0.39 \\
\hline
\end{tabular}

Note: * Statistically significant if $p<0.05, n-$ sample size, $g / d L-G r a m s$ per deciliter.

Pain intensity range according to the international analog scale of pain was observed in Group I and amounted to 3.5 $(\mathrm{IQR}=2-4)$, in Group II the indicator was $1.5(\mathrm{IQR}=1-3)$; in
Group III - $2.0 \quad(\mathrm{IQR}=1-2)$, respectively $(\mathrm{p}<0.001)$. All patients were activated 3-4 hours after surgery. During the first 24 hours, the mean daily need for analgesia in Group I was 
52.2 $\pm 16.1 \mathrm{mg}$ of Dexketoprofen, in Group II $-28.5 \pm 14.1 \mathrm{mg}$, in Group III $-27.5 \pm 13.8 \mathrm{mg}$. Where is no difference between patients totally tubeless mPNL with a safety thread and totally tubeless $\mathrm{mPNL}$ in analgesia, respectively $(\mathrm{p}=0.39)$ ? The $100 \%$ stone-free rate was observed in Group I in $95.5 \%(21)$, in Group II - in 100.0\% (20) and in Group III - in 100.0\% (21) patients, $\mathrm{p}=0.60$. Postoperative fever $(>37.9 \mathrm{oC})$ was observed in $2(9.1 \%)$ patients from Group $\mathrm{I}$, in $1(5.0 \%)$ patient of Group II, in Group III this complication was not observed, $\mathrm{p}=0.36$. Complications in the form of perforation of the pleural cavity, trauma to the abdominal organs and major vessels in patients of this study were not observed, due to the continuous combined ultrasound and fluoroscopic monitoring during renal collecting system puncture.

Only one patient required from Group II after transfer to the postoperative ward, 40 minutes after the surgery was completed had bleeding from the percutaneous tract. After manual holding pressure on the tract, there was evidence of ongoing bleeding from the tract and decision was made to place a nephrostomy catheter for hemostasis. Sonographic examination of the kidney showed moderate distention of the renal collecting system with the presence of blood clots. The patient was transferred to the treatment room and positioned in a lateral position with the affected side up. The percutaneous tract was injected with $10 \mathrm{ml}$ of a $2,0 \%$ lidocaine solution. The proximal and distal ends of the safety thread were loosened from ligatures on the skin and from the urethral catheter. The proximal and distal ends of the safety thread were held on tension with the help of an assistant. A Foley catheter (12Fr, with open end) was used as a nephrostomy tube, and this was placed over the distal (percutaneous) end of the safety suture and transcutaneously inserted into the renal cavity system under sonographic guidance. Once the tip of the catheter was visualized in the renal pelvis, the Foley catheter balloon was inflated to $1.5 \mathrm{ml}$ and the catheter was placed on mild traction. After 2-3 minutes of Foley catheter traction, bleeding through the nephrostomy tract ceased. Nephrostomy drainage was fixed to the skin. The tips of safety threads were secured to the urethral catheter and nephrostomy.

The following day, no bleeding was observed, but according to sonographic examination data, the presence of blood clots in the renal cavity system was noted. In order to prevent obstruction of the urinary tract, we decided to insert a ureteral JJ-stent. The patient was taken to endoscopic procedure suite and intravenous sedation (Propofol 1.0\%, $10 \mathrm{ml}$ ) was administered with the patient in a lithotomy position. The urethral tip of the safety thread was loosened from the urethral catheter and the latter was removed. Without the use of a cystoscope, under X-ray control, a ureteral catheter was inserted into the ureter in a retrograde fashion along a safety thread. The percutaneous tip of the safety thread was loosened from the nephrostomy drainage and the thread was removed. Retrograde pyelography was performed, a wire guide was passed through a ureteral catheter into the renal collecting system, followed by removal of the ureteral catheter and insertion of a ureteral JJ-stent under fluoroscopic guidance. The following day, the nephrostomy was removed, and the patient was discharged from the clinic. The ureteral stent was removed 14 days later. No bleeding recurrence was observed. The mean hospital stays in Group I was $28.4 \pm 9.5$ hours, in Group II $-27.6 \pm 11.7$ and in Group III $-25.1 \pm 5.2$ hours (no differences were found, $\mathrm{p}=0.39$ ).

\section{DISCUSSION}

Today, percutaneous nephrolithotomy remains the preferred procedure for treatment of kidney stones larger than 1.5-2.0 $\mathrm{cm}$ [3]. Since initial reports by Winfield et al. [14] nephrostomy drain was meant to provide mechanical hemostasis, adequate drainage of the renal collecting system, and prevent urinary extravasation into the paranephric tissue. However, presence of a nephrostomy tube has been shown to increase morbidity of the procedure due to increased postoperative pain and analgesic requirement. However, there's been a increasing acceptance of completing the surgery without a nephrostomy, and providing drainage of the upper urinary tract via a ureteral catheter or stent (tubeless) and in some cases completely without a drain (totally tubeless). Several studies have shown that in select patients' standard methods of completing PNL can be safely replaced by tubeless/totally tubeless ones [10;15-17].

Our study provides further evidence that in select cases tubeless /totally tubeless mPNLs is a safe and effective modality for treatment of nephrolithiasis, comparable both in terms of $100 \%$ SFR $(p=0.60)$ and in the level of complications: mean hemoglobin drops $(\mathrm{p}=0.62)$ and postoperative fever $(p=0.36)$. However, even if all conditions are met before performing a totally tubeless $\mathrm{mPNL}$, there remains a potential risk of bleeding from the percutaneous tract in the early postoperative period $[17 ; 18]$. Moreover, not all surgeons, especially those without significant experience in tubeless techniques, may not be willing to cross the psycho-emotional barrier to move away from traditional methods of completing PNL without nephrostomy drainage in the percutaneous tract due to the risk of postoperative complications. It is additionally explained by the fact that immediately after surgery there is a risk of blood clots formation, due to even clinically insignificant capillary bleeding, which in the postoperative period can cause obstructing clot to form in the ureter, and as a consequence lead to renal colic and leakage of urine through the nephrocutaneous tract. In this case, the lack of control over the parenchymal tract may require additional interventions (placement of a nephrostomy or ureteral JJstent), which ultimately increases the economic costs and treatment duration for patients. $[11 ; 13 ; 16]$.

Thus, in the initial stages of performing tubeless mPNL, we preferred the Shpall's tubeless mPNL technique [7], in which the parenchymal tract was under control. However, this technique assumes the presence of a ureteral JJ-stent in the urinary tract, which may be accompanied by discomfort in patients in the postoperative period. $[10 ; 15 ; 16]$. At the same 
time, the removal of the ureteral stent implies the removal of the extraction of the percutaneous sutures to the outside without cystoscopy, which also requires anesthesia of this procedure. The positive side of this technique, which allows continuous postoperative monitoring of the percutaneous canal, has helped us to offer modified completely tubeless $\mathrm{mPNL}$ technique with continuous controlling of the parenchymal canal. At the same time, the presence of a safety thread in the urinary tract does not cause postoperative discomfort in patients, which is comparable to the totally tubeless mPNL both in the level of postoperative discomfort and in the level of postoperative analgesia, in contrast to patients who underwent tubeless PNL with the installation of a ureteral JJ-stent $(<0.001)$. And of course, the removal of the safety thread does not require cystoscopy and analgesia.

\section{CONCLUSION}

This study shows benefits of performing totally tubeless PNL in terms of postoperative pain, and need for analgesia. Proposed modified procedure of totally tubeless PNL with the insertion of a safety thread offers the same benefits as a totally tubeless technique, while maintaining continuous control over the percutaneous tract and renal collecting system in case of bleeding. The technique may be helpful as an intermediate step for surgeons who are considering transition to a totally tubeless PCNL technique. However, it should be noted that a small number of observations are significant limiting factors for the final conclusion and recommendations for the use of this technique in wide clinical practice. Therefore, the continuation of further research on this issue remains an urgent task.

Our proposed procedure of conducting totally tubeless $\mathrm{mPNL}$ with a safety thread, provides the surgeon with constant control over the percutaneous tract, and allows for quick placement of a nephrostomy tube in case of bleeding or ureteral JJ-stent in case of ureteral obstruction, without transfer patient to the operating room. Our study is not without limitations. Specifically, the study is limited by the small number of patients studied. There were no statistically significant differences in demographic characteristics, the preoperative location of the stones and in the intraoperative results in the groups under study. At the same time, if surgery is completed with totally tubeless $\mathrm{mPNL}$ or totally tubeless mPNL with a safety thread, the level of postoperative pain decreases, the need for analgesia is less, which is explained by the absence of a ureteral JJ-stent, which can contribute to increase in lower urinary tract symptoms.

\section{ACKNOWLEDGMENT}

\section{Ethical Compliance}

The Department of Urology Inspection Commission, Shupyk National Healthcare University of Ukraine, has approved this study. All procedures performed in our study involving human participants were in accordance with the ethical standards of the institutional and/ or national research committee and Declaration of Helsinki (1964) and its later amendments or comparable ethical standards. For this type of study, formal consent is not required.

\section{Disclosure Statement}

This study has not received a single grant from funding agencies in the public, commercial, or non-profit sectors.

\section{Conflicts of Interest}

Authors have no conflict of interest to declare.

\section{References}

[1] P. Aarthy, M. Thangarasu, J. S. Prakash, D. Raghavan, N. Jain, A. Balakrishnan, M. Chandranathan, S. Bafna, R. Paul, N. Selvaraj, "Safety and efficacy of minipercutaneous nephrolithotomy as daycare procedure: a prospective observational study". African Journal of Urology, vol. 27, no. 1, article number 9, 2021.

[2] G. Zeng, C. Cai, X. Duan, X. Xu, H. Mao, X. Li, Y. Nie, J. Xie, J. Li, J. Lu, X. Zou, J. Mo, C. Li, J. Li, W. Wang, Y. Yu, X. Fei, X. Gu, J. Chen, X. Kong, J. Pang, W. Zhu, Z. Zhao, W. Wu, H. Sun, Y. Liu, J. D. la Rosette, "Mini percutaneous nephrolithotomy is a noninferior modality to standard percutaneous nephrolithotomy for the management of $20-40 \mathrm{~mm}$ renal calculi: A multicenter randomized controlled trial. European Urology, vol. 79, no. 1, pp. 114-121, 2021.

[3] C. Türk, A. Neisius, A. Petrik, 2020. Urolithiasis. European Association of Urology. Available: https://uroweb.org/guideline/urolithiasis/.

[4] J. E. Wickham, R. A. Miller, M. J. Kellett, S. R. Payne, "Percutaneous nephrolithotomy: one stage or two?" British Journal of Urology, vol. 56, 582-585, 1984.

[5] G. Bellman, R. Davidoff, J. Candela, J. Gerspach, S. Kurtz, L. Stout, "Tubeless percutaneous renal surgery". Journal of Urology, vol. 157, 1578-1582, 1997.

[6] J. Yew, G. Bellman, "Modified "tubeless" percutaneous nephrolithotomy using a tail stent". Urology, vol. 62, pp. 346-349, 2003.

[7] A. I. Shpall, A. R. Parekh, G. C. Bellman, "Modification of tubeless percutaneous nephrolithotomy: Anterograde stent with flank tether". Urology, vol. 68, pp. 880-882, 2006.

[8] M. Helal, T. Black, J. Lockhart, T. E. Figueroa, "The Hickman peel-away sheath: alternative for pediatric percutaneous nephrolithotomy". Journal of Endourology/Endourological Society, vol. 11, pp. 171172, 1997.

[9] S. Lahme, K. H. Bichler, W. L. Strohmaier, "Minimally invasive PCNL in patients with renal pelvic and calyceal stones". European Urology, vol. 40, no. 6, pp. 619-624, 2001.

[10] J. Alsmadi, J. Fan, W. Zhu, Z. Wen, G. Zeng, "The Influence of Super-Mini Percutaneous Nephrolithotomy on Renal Pelvic Pressure in Vivo". Journal of Endourology, vol. 32, no. 9, pp. 819-823, 2018.

[11]D. N. Thomas, W. Atallah, R. Chandhoke, J. N. Bamberger, M. Gupta, "Ultrasound-Guided Totally Tubeless Mini-Percutaneous Nephrolithotomy for a 
Completely Excluded Caliceal Diverticulum Associated with Challenging Anatomy". Journal of Endourology Case Reports, vol. 6, no. 3, pp. 121-123, 2020.

[12] M. R. Abdel Gawwad, A. T. Ozdemir, "Interactome Analysis and Docking Sites Prediction of (AtCHR8, AtCUL4 and AtERCC1/UVR7) Proteins in Arabidopsis Thaliana". Heritage and Sustainable Development, vol. 2, no. 1 , pp. 52-68, 2020.

[13]F. Karadeniz, Ö. E. Aydoğan, E. A. Kazanc1, E. Akdogan, "Design of a 4-DOF Grounded Exoskeletal Robot for Shoulder and Elbow Rehabilitation". Sustainable Engineering and Innovation, vol. 2, no. 1, pp. 41-65, 2020.

[14] I. Ligomina, V. Sokolyuk, S. Furman, D. Lisogurska, O. Lisogurska, "Clinical and Biochemical Status of Calves for D-Hypovitaminosis". Scientific Horizons, vol. 1, no. 86, pp. 89-95, 2020.

[15] A. Adilović, A. Karić, J. Šutković, "Brassica Oleracea L. Var. Acephala ABA Biosynthesis Genes (NCED2 and NCED3) in Silico Interactome Analysis". Heritage and Sustainable Development, vol. 1, no. 2, pp. 84-94, 2019.

[16]L. Horalskyi, O. Kovalchuk, I. Sokulskyi, "Pathamorphological Changes of Cats Pancreata Under Acute Pancreatitis". Scientific Horizons, vol. 8, no. 93, pp. 149-157, 2020.

[17]L. Quibo, G. Liang, L. Jie, "Total tubeless versus standard percutaneous nephrolithotomy: a meta-analysis". Minimally Invasive Therapy \& Allied Technologies, vol. 29, no. 2, pp. 61-69, 2020.

[18] S. W. Choi, K. S. Kim, J. H. Kim, "Totally tubeless versus standard percutaneous nephrolithotomy for renal stones: analysis of clinical outcomes and cost". Journal of Endourology, vol. 28, pp. 1487-1494, 2014.

[19] Y. Kanda, "Investigation of the freely available easy-touse software 'EZR' for medical statistics". Bone Marrow Transplant, vol. 48, pp. 452-458, 2013.

[20]D. O. Oseikhuemen, R. Saatchi, "Principal component analysis of the modified clinical test of sensory interaction in healthy adult humans". WSEAS Transactions on Biology and Biomedicine, vol. 17, pp. 125-142, 2020

[21]M. E. Omar Abdel-Salam, R. Eman Youness, A. S. Amany. A. Enayat Omara,"Oxidative stress and neuronal injury after cannabis and ketamine administration", WSEAS Transactions on Biology and Biomedicine, vol. 18, pp. 126-135, 2021

[22]K. S. Bisselou, G. Haynatzki, "Bias corrected Hlikelihood approach for joint models of longitudinal and survival data, with application to community acquired pneumonia", WSEAS Transactions on Biology and Biomedicine, vol. 18, pp. 119-125, 2021

\section{Author Contributions:}

Andriy I. Sahalevych: Conceptualization, Formal analysis and Writing - original draft.

Roman V. Sergiychuk: Investigation, Formal analysis and Writing - original draft and Writing - review \& editing.
Vladislav V. Ozhohin: Investigation, Formal analysis and Writing - original draft and Writing - review \& editing.

Andriy Yu. Khrapchuk: Investigation \& editing.

Yaroslav O. Dubovyi: Investigation, Formal analysis \& editing.

Olexander S. Frolov: Investigation, Formal analysis and Writing - original draft \& editing.

\section{Creative Commons Attribution License 4.0 (Attribution 4.0 International, CC BY 4.0)}

This article is published under the terms of the Creative Commons Attribution License 4.0 https://creativecommons.org/licenses/by/4.0/deed.en_US 